\title{
The role of aldehyde dehydrogenase-I (ALDHIAI) polymorphisms in harmful alcohol consumption in a Finnish population
}

\author{
Penelope A. Lind,' C.J. Peter Eriksson ${ }^{2 *}$ and Kirk C. Wilhelmsen ${ }^{3}$ \\ 'Department of Genetic Epidemiology, Queensland Institute of Medical Research, PO Royal Brisbane Hospital, QLD 4029, \\ Australia \\ ${ }^{2}$ Department of Mental Health and Alcohol Research, National Public Health Institute, P.O. Box 33, 00251 Helsinki, Finland \\ ${ }^{3}$ Department of Genetics and Neurology, Bowles Center for Alcohol Studies, University of North Carolina at Chapel Hill, NC \\ 27599, USA \\ *Correspondence to: Tel: +358 94744 8114; Fax: +358 94744 8133; E-mail: peter.eriksson@ktl.fi
}

Date received (in revised form): 16th May, 2008

\begin{abstract}
Liver cystolic aldehyde dehydrogenase I (ALDHIAI) has been previously associated with both alcohol dependence and alcohol consumption behaviour, and has been implicated in alcohol-induced flushing and alcohol sensitivity in Caucasians. The present study tested for association between ALDHIAI and alcohol consumption behaviour and susceptibility to problem drinking or alcohol dependence in Finnish cohorts of unrelated male subjects recruited from alcoholism clinical treatment facilities $(n=104)$ and from the general population $(n=20 \mathrm{I})$. All participants completed the Alcohol Use Disorder Identification Test (AUDIT) and were genotyped for eight single nucleotide polymorphisms (SNPs) within or flanking ALDHIAI. To test for association between alcohol consumption behaviour and these polymorphisms, we used generalised linear models and haplotypic analysis. Three SNPs were nominally associated ( $r s 348449, p=0.043$; rs610529, $p=0.013$; rs348479, $p=0.025$ ) with the quantitative AUDIT score, which evaluates alcohol consumption behaviour. Two-locus (rs6I0529-rs2288087) haplotype analysis increased the strength of association with AUDIT score $(p=0.00$ I5). Additionally, $r s 348449$ is highly associated with problem drinking (allelic odds ratio [OR] 7.87, 95 per cent confidence interval $[\mathrm{Cl}]$ $1.67-37.0 \mathrm{I})$ but due to the low minor allele frequency $(0.0 \mathrm{I}$ and 0.07 in controls and problem drinkers, respectively), more samples are required to validate this observation. Conversely, rs348479 ( $p=0.019)$ and rs610529 (allelic OR 0.65, 95 per cent $\mathrm{Cl} 0.43-0.98$; genotypic OR 0.32, 95 per cent $\mathrm{Cl} 0.12-0.84$ ) are implicated in alcohol dependence status. This study provides further evidence for a role for ALDHIAI in alcohol consumption behaviour, including problem drinking and possibly alcohol dependence, in our Finnish population.
\end{abstract}

Keywords: acetaldehyde, ALDHIAI, alcohol use, alcohol abuse, alcohol dependence, association, single nucleotide polymorphism

\section{Introduction}

Cystolic aldehyde dehydrogenase (ALDH1A1) has been associated with alcohol-induced flushing in Caucasians, and alcohol sensitivity and dependence, ${ }^{1,2}$ and functions in the detoxification of acetaldehyde, the first metabolite of ethanol oxidation. ${ }^{3}$ Additionally, ALDH1A1 is involved in the degradation of dopamine (DA) in the ventral 
tegmental area (VTA). ${ }^{4}$ This role in the regulation of DA degradation may be important in the development and maintenance of excessive alcohol consumption because VTA DA function can be ethanol induced via increased DA neurone firing, and the VTA lies within the mesolimbic system, which functions in reward, motivation and various substance disorders, including alcohol dependence. $^{5,6}$

Association between genetic variation in ALDH1A1 (on chromosome 9q21.13) and interindividual differences in the vulnerability to alcohol dependence and other alcohol-related phenotypes has been investigated in several studies. While there are three alternative splice patterns producing two ALDH1A1 isoforms, ${ }^{7}$ ALDH1A1 is highly conserved in humans. Two promoter polymorphisms have recently been correlated with the development of alcohol dependence in south-west Californian Indians ${ }^{8}$ and other populations. ${ }^{1,9}$ Spence and colleagues $^{1}$ sequenced the ALDH1A1 promoter region in Asian, Caucasian and African-American alcoholics and control subjects. They discovered two rare promoter polymorphisms, ALDH1A1*2 and $A L D H 1 A 1^{*} 3$ (only detected in African Americans). While no association was observed between $A L D H 1 A 1^{*} 2$ and alcohol dependence, there was a nominally significant difference in ALDH1A1*3 allelic frequency between alcoholics (6 per cent) and controls (0), and the ALDH1A1*3 polymorphism resulted in reduced ALDH1A1 expression. Ehlers et al. ${ }^{8}$ replicated this study in a south-west Californian Indian population of alcoholics and controls and observed a protective role for the $A L D H 1 A 1^{*} 2$ polymorphism, whereby individuals with an $A L D H 1 A 1^{*} 2$ allele reported lower rates of alcohol dependence, drank fewer drinks per session and reported a lower maximum number of drinks consumed in a 24-hour period. Conversely, the $A L D H 1 A 1^{*} 2$ alleles were later reported to be associated with increased development of alcohol dependency in Indo-Trinidadians. 9

Two other studies have reported genetic linkage in the $9 q 21$ region with phenotypic measures of alcohol consumption in predominantly Caucasian American samples from the Framingham Heart
Study (FHS). Bergen et al. ${ }^{10}$ observed nominally significant evidence of linkage in the $9 q 21.11$ region between three loci and a squareroot-transformed 'maximum alcohol consumed in a 24-hour period' variable, MAXAPD. Stronger evidence for linkage of MAXAPD on chromosome 9 was detected by $\mathrm{Ma}$ et al. ${ }^{11}$ with a maximal logarithm of the odds ratio (LOD) score of 2.27 in the region of ALDH1A1.

Due to the recently established role of $A L D H 1 A 1$ in alcohol-related phenotypes and the dual function of $A L D H 1 A 1$ in both the alcohol and DA metabolism pathways, we screened eight polymorphisms across the 52.8-kilobase (kb) ALDH1A1 gene for association with alcohol consumption patterns and alcohol dependence in our Finnish population. Alcohol dependence and problem drinking were determined by alcoholism treatment status and the Alcohol Use Disorder Identification Test (AUDIT), a measure of alcohol consumption behaviour.

\section{Materials and methods}

\section{Recruitment and assessment of subjects}

The Finnish subjects (with Finnish ethnicity) consisted of 104 men enlisted during clinical treatment for alcoholism and a sample of 201 male volunteers from the general population (including alcoholdependent and control subjects), either recruited through advertisements $(n=129)$ or drawn from the Finnish Population Register $(n=72)$ by the National Public Health Institute of Helsinki, Finland. All subjects were from the metropolitan area. Blood was obtained from all subjects and total genomic DNA was extracted using the PUREGENE ${ }^{\circledR}$ DNA Isolation Kit (Gentra, Inc., Minneapolis, MN, USA) according to the manufacturer's instructions.

Hazardous or harmful alcohol consumption behaviour and alcohol-related problems were assessed by the AUDIT. This instrument consists of ten items, which, when responded to in the affirmative, increase the likelihood that the subject is alcohol dependent. The questionnaire measures alcohol intake (quantity and frequency; items 1-3), 
dependence symptoms (items 4-6) and alcoholrelated problems (items $7-10$ ). All items are equally weighted and scored between 0 and 4 and the completed AUDIT is scored by summing the values for each item. The interpretation of the AUDIT, with respect to alcohol use disorders, has been well validated and reviewed by a number of groups. ${ }^{12-15}$ Babor et al. ${ }^{16}$ distinguished between hazardous and harmful drinking, whereby hazardous alcohol intake is indicative of an increased risk of harmful consequences for the user or others, and harmful alcohol use is defined as consumption behaviour that has resulted in physical or psychological damage. Additionally, Babor and colleagues ${ }^{14}$ recommend further evaluation for alcohol dependence for those who score 20 or higher. Accordingly, our study population was subdivided into four groups based upon total AUDIT score and history of alcohol dependence treatment: (1) control drinkers scored $\leq 12$ on the AUDIT but were not alcohol naïve $(n=105)$; (2) hazardous drinkers scored 13-19 on the AUDIT (excluded from further genetic analyses, $n=32$ ); (3) problem drinkers had an AUDIT score $\geq 20 \quad(n=64)$; and (4) treated alcoholics were recruited from alcohol treatment facilities $(n=104)$.

\section{Genotyping}

Since genotype data were not available from the International HapMap Project public database ${ }^{17}$ at the time this study was conceived (July 2003), eight single nucleotide polymorphisms (SNPs) in or near the ADLH1A1 locus were selected from the Applied Biosystems SNP database. ${ }^{18}$ SNPs were initially chosen to be evenly distributed across $A D L H 1 A 1$, including one SNP in both the $5^{\prime}$ and $3^{\prime}$ untranslated regions (UTRs), with an average inter-marker spacing of $10.7 \mathrm{~kb}$.

All primers, probes and reagents were purchased from Applied Biosystems Inc. (ABI; Foster City, CA, USA). To ensure quality control, DNA samples from cases and controls were randomly distributed across a 384-well plate. SNPs were genotyped using TaqMan ${ }^{\mathrm{TM}}$ fluorescence $5^{\prime}$ exonuclease technology. Each $5 \mu \mathrm{l}$ experiment contained $25 \mathrm{ng}$ genomic DNA, 1.6X TaqMan assay primer/probe mix, 1X PCR buffer A, $2.5 \mathrm{mM} \mathrm{MgCl}_{2}, 250 \mu \mathrm{M}$ deoxyribonucleotide triphosphates (dNTPs) and 0.5 U AmpliTaq Gold polymerase. Thermocycling was performed as recommended by ABI using a Dual 384-Well GeneAmp® PCR System 9700 cycler and cycling conditions as follows: an initial denaturation stage at $95^{\circ} \mathrm{C}$ for 12 minutes, then 40 cycles of $95^{\circ} \mathrm{C}$ for 15 seconds and $60^{\circ} \mathrm{C}$ for one minute, followed by storage at $4^{\circ} \mathrm{C}$ in the dark. Genotypes were determined on an ABI 7900HT Fast Real-Time PCR System using the allelic discrimination mode.

\section{Statistical analysis}

All polymorphisms were first assessed to determine if the observed genotype frequencies were consistent with Hardy-Weinberg proportions using Pearson chi-square tests within Stata (version 8.1; Stata Corporation, College Station, TX, USA). Pair-wise marker-marker linkage disequilibrium (LD) was assessed in Haploview (version 4.0). ${ }^{19}$ To test for association between each SNP and the continuously distributed 'AUDIT score', we performed analysis of variance models (ANOVA) using PROC GLM within the SAS software system (version 8.0; SAS Institute, Cary, NC, USA). Genotype was first tested for general association (no mode of inheritance assumption) using a two-degrees of freedom F-test. Further testing of the SNP effects assuming genetic modes of inheritance for that SNP were then performed. For dominant or recessive genetic transmission models, a single indicator variable (eg $\mathrm{I}_{\mathrm{dom}}$ is an indicator variable taking the value 1 if an individual has genotype $1 / 2$ or $2 / 2$, and 0 otherwise) was used. A variable taking the values -1 for genotype $1 / 1$; 0 for genotype $1 / 2$; and 1 for genotype $2 / 2$ was used to test for additive genetic effects.

In addition, odds ratios (OR) and 95 per cent Wald confidence intervals (95 per cent CI) were estimated in Stata 8.1 for both the allelic and genotypic distribution by dividing the dataset into controls $(n=105$, AUDIT score $\leq 12)$, problem drinkers $(n=64$, AUDIT score $\geq 20)$ and treated alcoholics $(n=104)$. Genotype and allele 
Table I. Alcohol Use Disorder Identification Test (AUDIT) response, mean and standard deviation per item, in the Finnish control drinkers, problem drinkers and treated alcohol-dependent groups, based on AUDIT score and/or alcohol treatment status

\begin{tabular}{|c|c|c|c|c|c|}
\hline Item & Assessment & $\begin{array}{l}\text { AUDIT item } \\
\text { description }^{\mathrm{a}}\end{array}$ & Controls $^{b}$ & $\begin{array}{l}\text { Problem } \\
\text { drinkers }^{c}\end{array}$ & $\begin{array}{l}\text { Treated } \\
\text { alcoholics }^{d}\end{array}$ \\
\hline- & Subjects(n) & - & 105 & 64 & 104 \\
\hline - & $\begin{array}{l}\text { Clinically treated } \\
\text { for alcoholism }\end{array}$ & - & 0 & 0 & $100 \%$ \\
\hline I & $\begin{array}{l}\text { Hazardous alcohol } \\
\text { consumption }\end{array}$ & $\begin{array}{l}\text { Frequency of } \\
\text { drinking days }\end{array}$ & $2.5 \pm 1.0$ & $3.4 \pm 0.8$ & $3.1 \pm 1.0$ \\
\hline 2 & $\begin{array}{l}\text { Hazardous alcohol } \\
\text { consumption }\end{array}$ & $\begin{array}{l}\text { Average number of } \\
\text { drinks per session }\end{array}$ & $I . I \pm I . I$ & $3.4 \pm 10.8$ & $3.4 \pm 1.1$ \\
\hline 3 & $\begin{array}{l}\text { Hazardous alcohol } \\
\text { consumption }\end{array}$ & $\begin{array}{l}\text { Frequency of six or } \\
\text { more drinks }\end{array}$ & $1.4 \pm 0.8$ & $3.4 \pm 0.7$ & $3.0 \pm 1.0$ \\
\hline 4 & $\begin{array}{l}\text { Dependence } \\
\text { symptoms }\end{array}$ & $\begin{array}{l}\text { Impaired control } \\
\text { over drinking }\end{array}$ & $0.1 \pm 0.3$ & $2.6 \pm 1.2$ & $2.3 \pm 1.4$ \\
\hline 5 & $\begin{array}{l}\text { Dependence } \\
\text { symptoms }\end{array}$ & $\begin{array}{l}\text { Increased salience } \\
\text { of drinking }\end{array}$ & $0.1 \pm 0.3$ & $2.0 \pm 1.2$ & $2.3 \pm 1.3$ \\
\hline 6 & $\begin{array}{l}\text { Dependence } \\
\text { symptoms }\end{array}$ & Morning drinking & $0.1 \pm 0.3$ & $2.6 \pm 1.3$ & $2.7 \pm 1.3$ \\
\hline 7 & $\begin{array}{l}\text { Alcohol-related } \\
\text { problems }\end{array}$ & Guilt after drinking & $0.5 \pm 0.6$ & $2.9 \pm 1.2$ & $2.7 \pm 1.4$ \\
\hline 8 & $\begin{array}{l}\text { Alcohol-related } \\
\text { problems }\end{array}$ & $\begin{array}{l}\text { Alcohol-related } \\
\text { blackouts }\end{array}$ & $0.4 \pm 0.5$ & $2.4 \pm 1.2$ & $2.4 \pm 1.3$ \\
\hline 9 & $\begin{array}{l}\text { Alcohol-related } \\
\text { problems }\end{array}$ & $\begin{array}{l}\text { Alcohol-related } \\
\text { injuries }\end{array}$ & $0.2 \pm 0.7$ & $2.2 \pm 1.5$ & $2.6 \pm 1.5$ \\
\hline 10 & $\begin{array}{l}\text { Alcohol-related } \\
\text { problems }\end{array}$ & $\begin{array}{l}\text { Others concerned } \\
\text { about drinking }\end{array}$ & $0.0 \pm 0.4$ & $3.4 \pm 0.9$ & $3.6 \pm 0.9$ \\
\hline - & $\begin{array}{l}\text { Hazardous/harmful } \\
\text { alcohol use }\end{array}$ & $\begin{array}{l}\text { AUDIT score (sum } \\
\text { of items) }\end{array}$ & $6.3 \pm 3.1$ & $28.3 \pm 5.4$ & $27.7 \pm 8.4$ \\
\hline
\end{tabular}

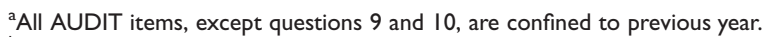

${ }^{\mathrm{b}}$ The Finnish population consists of alcoholic subjects recruited from alcohol treatment facilities $(n=104)$ and volunteer subjects from the general population that includes control drinkers $(n=105)$, hazardous drinkers $(n=32)$ and problem drinkers, as defined by the AUDIT $(n=64)$. Control drinkers had an AUDIT score $\leq 12$ and had consumed alcohol in the previous year.

cProblem drinkers have an AUDIT score $\geq 20$ but were not recruited from alcohol treatment facilities.

${ }^{\mathrm{d}}$ Treated alcoholics were recruited from alcohol treatment facilities.

${ }^{\mathrm{e}} \mathrm{An}$ AUDIT score $\geq 12$ indicates a pattern of hazardous consumption, while an AUDIT score $\geq 20$ suggests that the subject is alcohol dependent. ${ }^{13}$

distributions for the problem drinkers and treated alcoholic subjects were each compared with those in the controls as the reference group. Genotypic odds ratios were calculated by collapsing two genotypes (one heterozygous and one homozygous group) into one, and comparing the collapsed genotype count with that of the count of the remaining homozygous genotype.
To test for haplotypic association between the markers and AUDIT score, a generalised linear model for the quantitative trait 'AUDIT score' was run in Qtphase manufacturers. Qtphase is part of the UNPHASED ${ }^{20}$ suite of programs for association analysis of multilocus haplotypes (an expectationmaximisation [E-M] algorithm is used to deal with uncertain haplotypes) from unphased genotype 


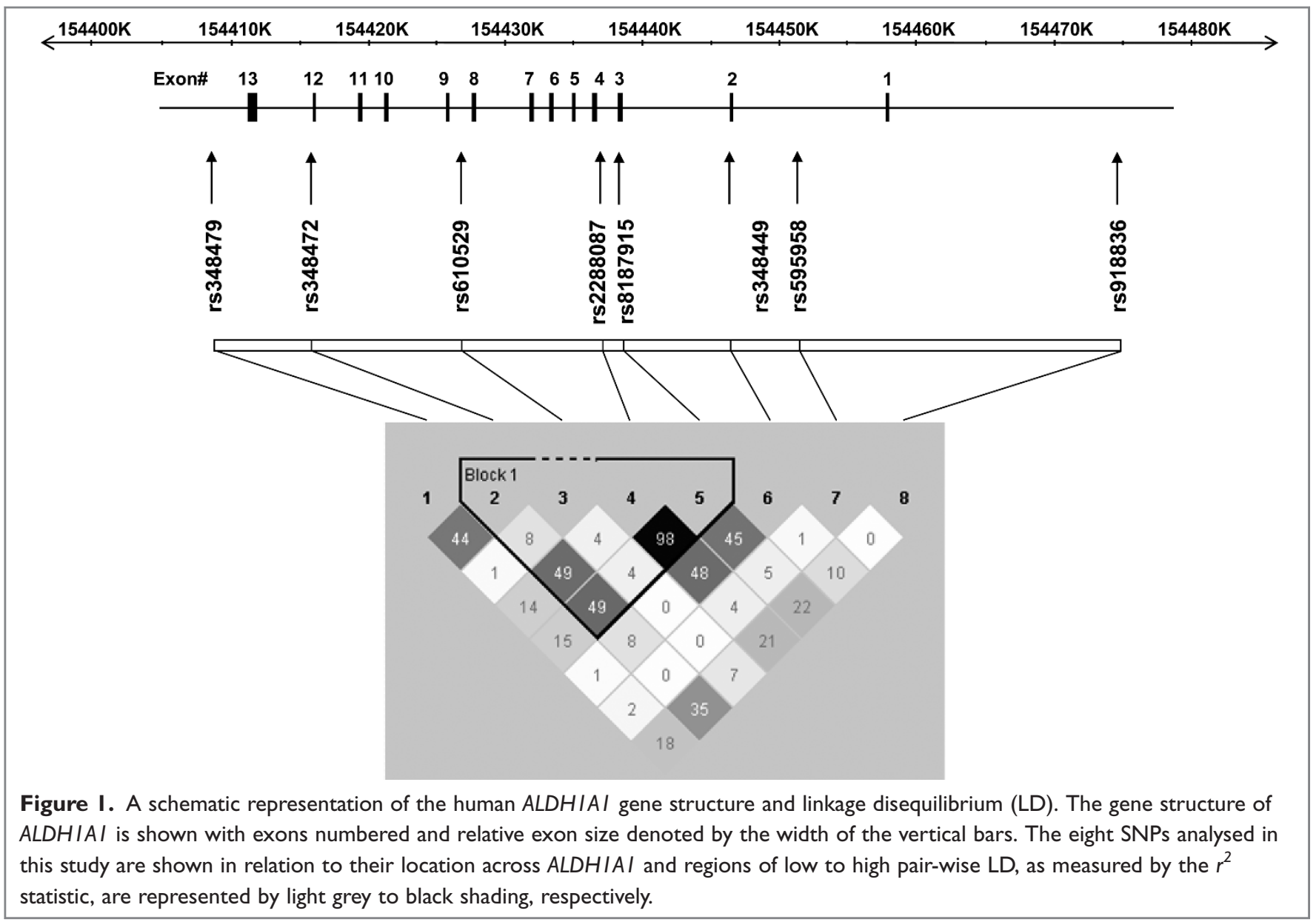

data. $^{21}$ All chi-square statistics and $p$-values are presented without correction for multiple testing.

\section{Results}

A total of 305 male participants provided blood samples and completed the AUDIT questionnaire. The mean age of the study population was $45.1 \pm$ 11.1 years, ranging from 21 to 75 years. While the average age of the treated alcoholics (44.3 years), problem drinkers (47.5 years) and control groups (44.2 years) at the time of interview did not differ significantly $(p>0.05)$, the average weight $(\mathrm{kg}, p=0.026)$ but not body mass index $\left(\mathrm{kg} / \mathrm{m}^{2}\right.$, $p=0.19)$ of the treated alcoholics $(77.6 \mathrm{~kg}$, $\left.24.7 \mathrm{~kg} / \mathrm{m}^{2}\right)$ and control $\left(81.3 \mathrm{~kg}, 25.3 \mathrm{~kg} / \mathrm{m}^{2}\right)$ groups did. Among the general population $(n=$ 201), 15.9 per cent displayed hazardous patterns of alcohol consumption and 31.8 per cent scored $\geq 20$ on the AUDIT and were classified in our analyses as problem drinkers (since they generally experienced both dependence symptoms and alcoholrelated problems) and were also included in the problem/dependent group.

The problem drinkers and treated alcoholics had marked differences $(p<0.0001)$ in their mean responses for all ten AUDIT items compared with the controls (Table 1). Although all groups tended to consume alcoholic beverages on a weekly basis (item 1; 55 per cent of controls and an average of 77 per cent for the problem and dependent drinkers), the majority (82 per cent) of problem drinkers and treated subjects consumed an average of six or more drinks per session (item 2) compared with 13 per cent of controls (on average, the control group imbibed three or four drinks per session). This trend was similar for the frequency with which subjects consumed six or more drinks 


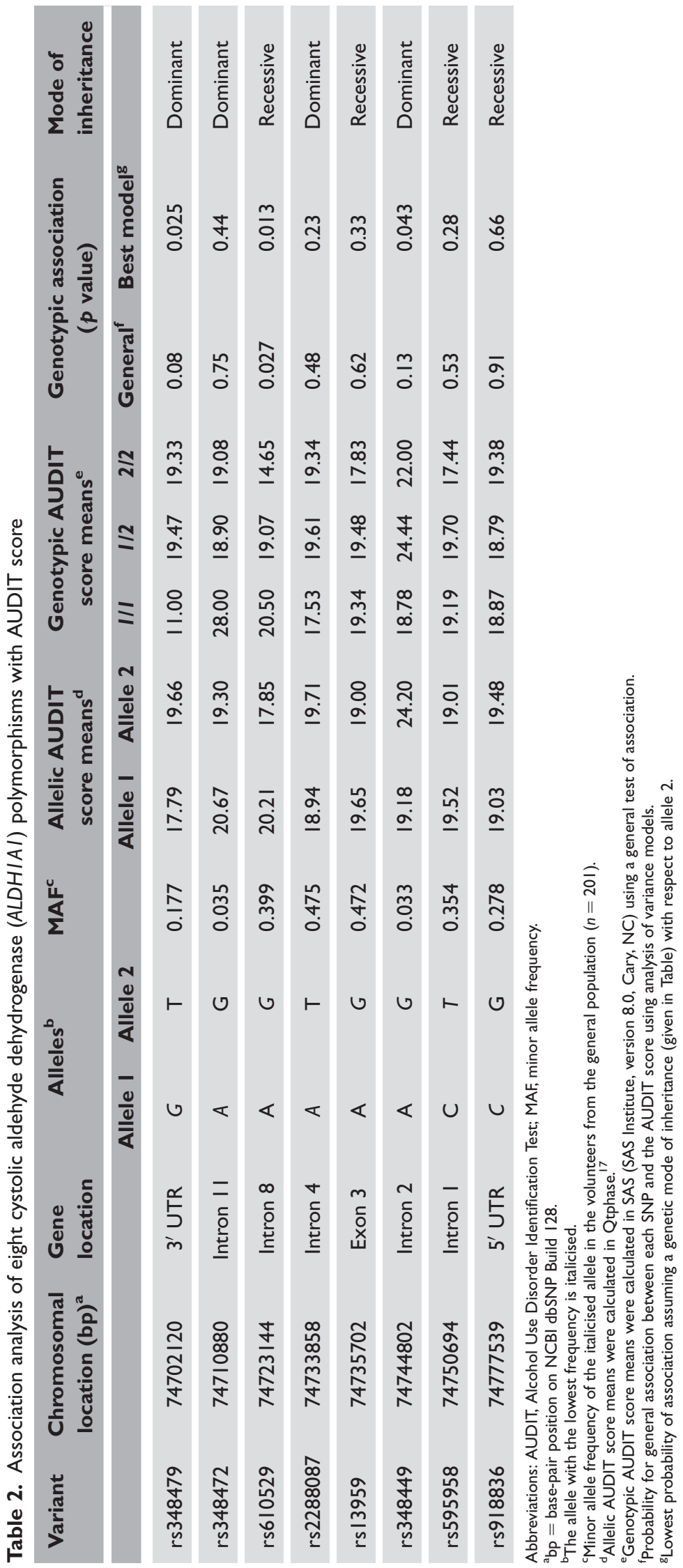




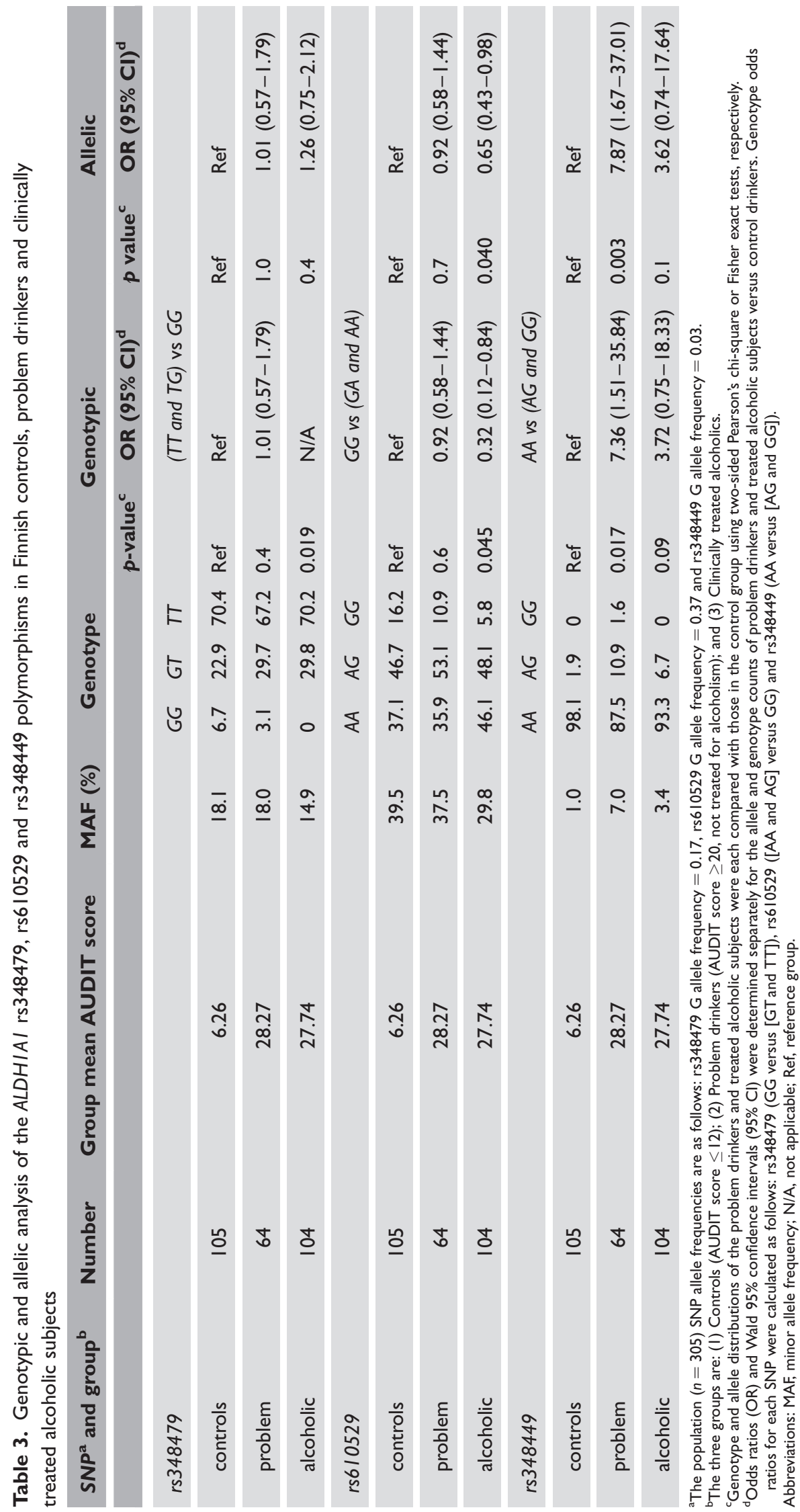




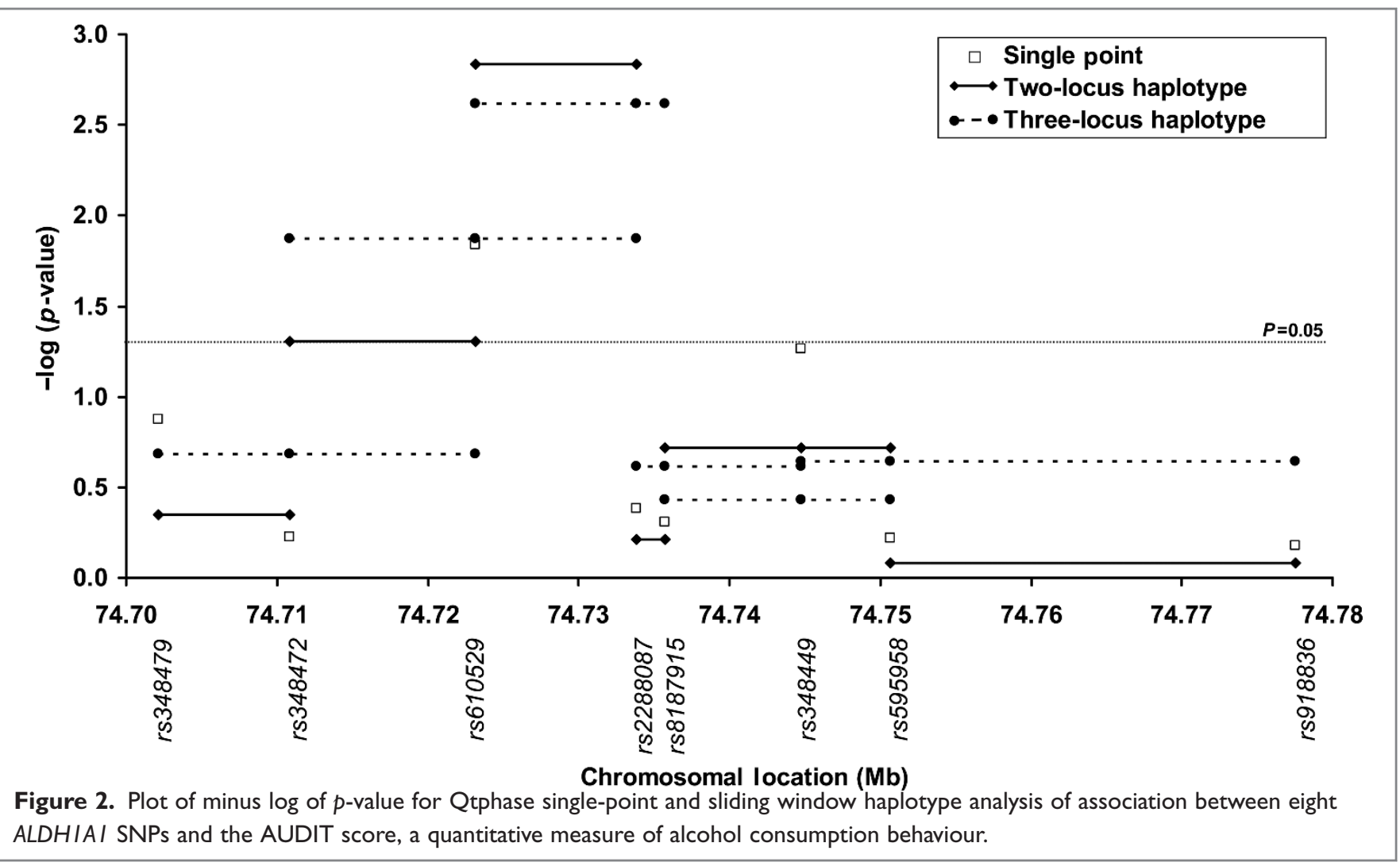

per session (item 3). While these significant differences in alcohol consumption behaviour (items 1-3) were observed between the control and problem/treated groups, an unambiguous distinction from the controls was seen with respect to those items related to dependence symptoms (items 4-6) and alcohol-related problems (items $7-10)$. For these seven items, the mean response was $\leq 0.5$ and $\geq 2.2$ for controls and problem/ treated groups, respectively, which on a 'occurs on a monthly or more' basis represents an affirmative response by $\leq 2$ per cent and $\geq 63$ per cent of the respective group subjects.

The physical locations of the eight SNPs across the $A L D H 1 A 1$ gene are schematically presented in Figure 1. Frequencies of the $A L D H 1 A 1$ genotypes were in general in Hardy-Weinberg equilibrium (HWE), except for rs595958 in intron 1 ( $p=$ $0.0008)$, and are listed in Table 2. Minor allele frequencies within the control sample were similar to those observed in the Centre d'Etude du Polymorphisme Humain (CEPH) sample (data not shown) of the International HapMap Project. ${ }^{22}$ The extent of LD between the eight SNPs is presented in Figure 1. LD was strongest between markers rs610529 (intron 8) and rs13959 (exon 3), with one haplotype block structure detected by Haploview $^{19}$ between these SNPs. The effective number of independent SNPs was 6.0, as determined by single nucleotide polymorphism spectral decomposition (SNPSpD). ${ }^{23,24}$

We observed nominally significant differences between the genotypic and/or allelic frequencies and mean AUDIT scores for three (rs348479 in the $3^{\prime}$ UTR, rs610529 in intron 8 and rs348449 in intron 2) of the eight SNPs genotyped in ALDH1A1. The genotypic distribution and allelic frequencies of the three SNPs are presented in Table 3.

The rs348479 $\mathrm{T}$ allele was more frequent in subjects with higher AUDIT scores, where the mean AUDIT scores observed for the rs348479 $\mathrm{G}$ and T alleles were 17.8 and 19.7 , respectively (Table 2) and the frequency of the T allele was 82 per cent in 
controls compared with 85 per cent (Table 3) in the treated alcoholic drinkers. In the analysis of the entire sample $(n=305)$, the association of the T allele with the AUDIT score approached nominal significance ( $p=0.08$ for general association) and the estimated genotypic AUDIT score means were suggestive of a dominant genetic model ( $p=$ $0.025)$ with respect to the $\mathrm{T}$ allele, where the AUDIT score is highest in those individuals with one or two copies of the $\mathrm{T}$ allele compared with individuals with two copies of the $G$ allele (mean AUDIT score of 11.0, 19.5 and 19.3 with zero, one and two rs348479 $\mathrm{T}$ alleles, respectively). The rs348479 genotypic frequencies were significantly different between the controls and treated alcoholics $(p=0.019)$; however, case-control odds ratio calculations did not indicate significant allele frequency differences between the controls and treated alcoholics (Table 3). Furthermore, the absence of significant allelic and genotypic differences between the controls and problem drinkers may indicate that rs348479 is involved in risk for alcohol dependence rather than increased or problem alcohol consumption. Nonetheless, the results of this study should also be interpreted in the context of multiple testing, since, correcting for the number of independent SNPs alone, a Bonferroni-corrected $p$-value of $0.0083(0.05 / 6)$ would be required for an association to be declared significant.

The rs610529 polymorphism in intron 8 was associated $(p=0.027$ and $p=0.013$ for the general and best model of association, which is based upon a recessive mode of inheritance for the $G$ allele) with the AUDIT score. The genotypic AUDIT score means were 20.5, 19.1 and 14.7 for zero, one and two rs610529 $\mathrm{G}$ alleles, respectively (Table 2). Although there were no significant differences in allelic frequencies between control and problem drinkers $(\mathrm{OR}=0.92,95$ per cent CI $0.58-1.44)$, treated alcoholics were approximately 1.5 times less likely to have the $\mathrm{G}$ allele $(\mathrm{OR}=0.65,95$ per cent CI 0.43-0.98) and three times more likely to be either a homozygous AA or a heterozygous AG $(\mathrm{OR}=0.32$, 95 per cent CI 0.12-0.84) (Table 3). As with rs348479, these analyses provide some evidence for an association between rs6105249 and risk for alcohol dependence rather than problematic alcohol consumption behaviour.

The rs348449 polymorphism was nominally associated $(p=0.043$ for the dominant model of inheritance; $p=0.13$ for general association) where the AUDIT score genotypic means were 18.9, 24.4 and 22.0 for zero, one and two G alleles, respectively (Table 2). Significant differences in both the rs348449 genotypic and allelic frequencies were detected between the controls and the problem drinkers (Table 3 ). The frequency of the minor allele $G$ was 1 per cent in controls compared with 7 per cent in the problem drinkers and 3.4 per cent in treated alcoholics. No G/G homozygotes were observed in the control and treated alcoholics groups. Problem drinkers were 7.8 times more likely to have the rs348449 $\mathrm{G}$ allele $(\mathrm{OR}=$ 7.87, 95 per cent CI 1.67-37.01, $p=0.003$ ) and 7.4 times more likely to have the GG or GA genotype $(p=0.017)$. By contrast, the allelic and genotypic distributions in the treated alcoholics and control groups were not significantly different. Therefore, rs348449 may play a role in the consumption of high levels of alcohol (which can lead to dependence symptoms and alcohol-related problems) rather than the development of alcohol dependence.

Since the age of the control subjects ranged between 24 and 69 years, it is possible that a subset of the younger controls could develop alcohol dependence later in life. Therefore, we divided the controls into an older cohort, aged over 40 years $(n=66)$, and repeated the allelic and genotypic analyses. With each SNP, we observed the same direction of, and similar levels of, association (data not shown).

Two- and three-locus haplotypic association between ALDH1A1 SNP genotypes and the quantitative AUDIT score is graphically presented in Figure 2. Using Qtphase and a sliding window approach, the significance of association increased from $p=0.014$ for the single rs610529 SNP analysis to 0.0015 (likelihood ratio statistic [LRS] $=$ 15.47, degrees of freedom $[\mathrm{DF}]=3$ ) for rs610529-rs2288087 haplotype analysis, where the 
two SNPs are in high $\operatorname{LD}\left(D^{\prime}=0.97, r^{2}=0.60\right)$. Three common haplotypes $(>1$ per cent frequency) were observed, totalling 99.4 per cent of the observed haplotypes. Two common haplotypes, '1-1' (10.4 per cent frequency) and '1-2' (35.9 per cent frequency) had mean AUDIT scores of 23.42 $\left(\chi^{2}=8.37, p=0.0038\right)$ and $17.64\left(\chi^{2}=7.3, p=\right.$ $0.0070)$, respectively. The third common haplotype ('2-1', 53.1 per cent) was not significantly associated $\left(\chi^{2}=0.4, p=0.55\right)$ with the AUDIT score (mean score $=19.59)$. The addition of a third neighbouring SNP, rs13959, did not increase the strength of association $(p=0.0024)$.

\section{Discussion}

Previous linkage and association studies of alcohol consumption or dependence measures have implicated the ALDH1A1 gene. ${ }^{1,2,8,10,11}$ Although the majority of alcohol studies related to the aldehyde dehydrogenase family focus on ALDH2, particularly in Asian populations, ${ }^{25,26}$ recent studies ${ }^{1,8,9}$ are shining light on the role that ALDH1A1 may play in several human alcohol-related traits. In the present study, we analysed eight SNPs located across $A L D H 1 A 1$ (including one SNP in both the $5^{\prime}$ and $3^{\prime}$ UTRs) for an association with alcohol dependence status (using case-control methodology) and the quantitative AUDIT score in our male Finnish population.

A 1992 study of Finnish drinking habits that utilised the AUDIT questionnaire determined that 22 per cent of males (26 per cent of men aged 30-49) who completed the AUDIT displayed hazardous alcohol consumption (alcohol consumption indicative of an increased risk of harmful consequences for the user or others) and 42 per cent usually drank seven or more units of alcohol per session (item 2), and concluded that in the Finnish culture there was an intoxication-seeking nature to alcohol consumption. ${ }^{27}$ In 2000 , the 12 -month prevalence of alcohol dependence and alcohol use disorders in a representative sample of male Finnish adults $(>30$ years) were 6.5 per cent and 7.3 per cent, respectively. $^{28}$ In this study, 15.9 per cent of subjects that were not recruited from alcoholism treatment facilities had experienced hazardous or harmful alcohol consumption in the previous year (AUDIT score of 12-19), which is similar to the findings of Holmila, ${ }^{27}$ while 31.8 per cent were categorised as problem drinkers (AUDIT score $\geq 20$ ). It should be noted, however, that alcohol-dependent and control drinkers were both specifically recruited in the advertisement campaign and, therefore, the problem-drinking group may have contained a percentage of alcohol-dependent individuals.

Our findings suggest that both alcohol consumption behaviour and alcohol dependence status are influenced by genetic variation in $A L D H 1 A 1$. The results from ANOVA analyses demonstrate that two intronic SNPs (rs610529 and rs348449) and one $3^{\prime}$ UTR SNP (rs348479) in ALDH1A1 are nominally $(p=0.01-0.04)$ associated with alcohol consumption behaviour, as described by the AUDIT (Table 2). While rs348479 and rs610529 are associated with risk of alcohol dependence rather than problem drinking, rs348449 in intron 2 influences harmful alcohol consumption behaviour that leads to alcohol-related problems and alcohol dependence symptoms (as determined by odds ratio and $\chi^{2}$ contingency analyses of allele and genotype frequencies in control and problem drinkers).

Although the allelic and genotypic distribution of rs348449 was not significantly different between controls and treated alcohol-dependent subjects, it may play a role in alcohol dependence. If Babor et al. ${ }^{14}$ are correct in their assertion that an AUDIT score $\geq 20$ indicates alcohol dependence, rs348449 may be associated with the transition from harmful alcohol consumption to alcohol dependence: the mean AUDIT scores were 19.2 and 24.2 for the A and $\mathrm{G}$ alleles, respectively, and on average a subject's AUDIT score increased from 18.8 to 24.4 and 22.0 with zero, one and two $G$ alleles, respectively. Additionally, Finns with a $G$ allele were 5.2-fold more likely to have been treated for alcohol dependence or have scored $\geq 20$ on the AUDIT. We have reservations with respect to the validity of the observed associations between this SNP and the two alcohol-related phenotypes, however; the low minor allele frequency (MAF) in the three drinking 
groups (1-7 per cent) and the absence of $G / G$ homozygotes among the control and treated alcohol dependent drinkers means that there is a high risk of false-positive finding with this SNP.

The three SNPs that demonstrated association with alcohol consumption behaviour in this study have not previously been reported in the literature and their functionality is unclear; they are neither coding SNPs nor are they near alternative splice sites. Nonetheless, we believe that at the genotypic and/or allelic level, each SNP may influence transitions in drinking patterns and behaviour in our population, particularly the progression from problem to alcohol-dependent drinker. For example, the genotypic AUDIT score means of the $3^{\prime}$ UTR SNP, rs348479, are 11.00 (these subjects are classified as control drinkers) and 19.33 (bordering between harmful and problem drinkers) for $\mathrm{G} /$ $\mathrm{G}$ and $\mathrm{T} / \mathrm{T}$ homozygotes, respectively. Furthermore, the three SNPs are in moderate LD $\left(D^{\prime}=0.4-0.6\right)$, so it may be possible that the SNPs are in strong LD with an as yet ungenotyped polymorphism that is causal with respect to these alcohol-related phenotypes. An important question, with regard to the relevance of the present data, is the functionality of these polymorphisms; this needs to be settled in future studies.

In conclusion, while the associations detected in the present study are not significant after taking into account multiple testing, multiple studies reporting associations between $A L D H 1 A 1$ and alcohol-related phenotypes have been published recently, and our results provide further evidence for a role of $A L D H 1 A 1$ in the risk for problem alcohol consumption behaviour and possibly alcohol dependence. With this in mind, our findings need to be replicated in a larger Finnish or Caucasian population and a systematic search for, and genotyping of, additional $A L D H 1 A 1$ variants that influence alcohol-related phenotypes is warranted.

\section{Acknowledgments}

The authors wish to thank Mrs Hilkka Salohalla, Mrs Tuula Mäkelä and Mrs Lolan Lindroos for their excellent technical assistance. This study was supported in part by funds provided by the State of California for medical research on alcohol and substance abuse through the University of California, San Francisco.

\section{References}

1. Spence, J.P., Liang, T., Eriksson, C.J.P. et al. (2003), 'Evaluation of aldehyde dehydrogenase 1 promoter polymorphisms identified in human populations', Alcohol Clin. Exp. Res. Vol. 27, pp. 1389-1394.

2. Yoshida, A., Dave, V., Ward, R.J. and Peters, T.J. (1989), 'Cytosolic aldehyde dehydrogenase (ALDH1) variants found in alcohol flushers', Ann. Hum. Genet. Vol. 53, pp. 1-7.

3. Quertemont, E. (2004), 'Genetic polymorphism in ethanol metabolism: Acetaldehyde contribution to alcohol abuse and alcoholism', Mol. Psychiatry Vol. 9, pp. 570-581.

4. Galter, D., Buervenich, S., Carmine, A. et al. (2003), 'ALDH1 mRNA: Presence in human dopamine neurons and decreases in substantia nigra in Parkinson's disease and in the ventral tegmental area in schizophrenia', Neurobiol. Dis. Vol. 14, pp. 637-647.

5. Balter, M. (1996), 'New clues to brain dopamine control, cocaine addiction', Science Vol. 271, pp. 909.

6. White, FJ. and Kalivas, P.W. (1998), 'Neuroadaptations involved in amphetamine and cocaine addiction', Drug Alcohol Depend. Vol. 51, pp. $141-153$.

7. http://www.ncbi.nih.gov/IEB/Research/Acembly/index.html.

8. Ehlers, C.L., Spence, J.P., Wall, T.L. et al. (2004), 'Association of ALDH1 promoter polymorphisms with alcohol-related phenotypes in southwest California Indians', Alcohol Clin. Exp. Res. Vol. 28, pp. 1481-1486.

9. Moore, S., Montane-Jaime, K., Shafe, S. et al. (2007), 'Association of ALDH1 promoter polymorphisms with alcohol-related phenotypes in Trinidad and Tobago', J. Stud. Alcohol Drugs Vol. 68, pp. 192-196.

10. Bergen, A.W., Yang, X.R., Bai, Y. et al. (2003), 'Genomic regions linked to alcohol consumption in the Framingham Heart Study', BMC Genet Vol. 4 (Suppl. 1), p. S101.

11. Ma, J.Z., Zhang, D., Dupont, R.T. et al. (2003), 'Mapping susceptibility loci for alcohol consumption using number of grams of alcohol consumed per day as a phenotype measure', BMC Genet. Vol. 4 (Suppl. 1), p. S104.

12. Reinert, D.F. and Allen, J.P. (2002), 'The Alcohol Use Disorders Identification Test (AUDIT): A review of recent research', Alcohol Clin. Exp. Res. Vol. 26, pp. 272-279.

13. Reid, M.C., Fiellin, D.A. and O'Connor, P.G. (1999), 'Hazardous and harmful alcohol consumption in primary care', Arch. Intern. Med. Vol. 159, pp. 1681-1689.

14. Babor, T.F., Higgins-Biddle, J.C., Saunders, J.B. and Monteiro, M.G. (2002), AUDIT: The Alcohol Use Disorders Identification Test, Guidelines for Use in Primary Care (2nd edn), World Health Organization, Geneva, Switzerland

15. Saunders, J.B., Aasland, O.G., Babor, T.F. et al. (1993), 'Development of the Alcohol Use Disorders Identification Test (AUDIT): WHO Collaborative Project on Early Detection of Persons with Harmful Alcohol Consumption - II', Addiction Vol. 88, pp. 791-804.

16. Babor, T.F., De La Fuente, J.R., Saunders, J.B. and Grant, M. (1989), AUDIT: The Alcohol Use Disorders Identification Test: Guidelines for Use in Primary Health Care (1st edn), World Health Organization, Geneva, Switzerland.

17. http://www.hapmap.org.

18. http://www.appliedbiosystems.com

19. Barrett, J.C., Fry, B., Maller, J. and Daly, M.J. (2005), 'Haploview: Analysis and visualization of LD and haplotype maps', Bioinformatics Vol. 21, pp. 263-265.

20. http://www.hgmp.mrc.ac.uk/ fdudbrid/software/unphased/.

21. Dudbridge, F. (2003), 'Pedigree disequilibrium tests for multilocus haplotypes', Genet. Epidemiol. Vol. 25, pp. 115-121. 
22. The International HapMap Consortium (2003), 'The international HapMap project', Nature Vol. 426, pp. 789-796.

23. http://gump.qimr.edu.au/general/daleN/SNPSpD/.

24. Nyholt, D.R. (2004), 'A simple correction for multiple testing for singlenucleotide polymorphisms in linkage disequilibrium with each other', Am. J. Hum. Genet. Vol. 74, pp. 765-769.

25. Chen, W.J., Loh, E.W., Hsu, Y.P. et al. (1996), 'Alcohol-metabolising genes and alcoholism among Taiwanese Han men: Independent effect of ADH2, ADH3 and ALDH2', Br. J. Psychiatry Vol. 168, pp. $762-767$.
26. Higuchi, S., Matsushita, S., Muramatsu, T. et al. (1996), 'Alcohol and aldehyde dehydrogenase genotypes and drinking behavior in Japanese', Alcohol Clin. Exp. Res. Vol. 20, pp. 493-497.

27. Holmila, M. (1995), 'Intoxication and hazardous use of alcohol: Results from the 1992 Finnish Drinking Habits Study', Addiction Vol. 90, pp. 785-792.

28. Pirkola, S.P., Isometsa, E., Suvisaari, J. et al. (2005), 'DSM-IV mood-, anxiety- and alcohol use disorders and their comorbidity in the Finnish general population - Results from the Health 2000 Study', Soc. Psychiatry Psychiatr. Epidemiol. Vol. 40, pp. 1-10. 